\title{
Solid-phase extraction of betanin and isobetanin from beetroot extracts using a dipicolinic acid molecularly imprinted polymer
}

Sofia Nestora, Franck Merlier, Elise Prost, Karsten Haupt, Claire Rossi* \& Bernadette Tse Sum Bui* Sorbonne Universités, Université de Technologie de Compiègne, CNRS Enzyme and Cell Engineering Laboratory, Rue Roger Couttolenc, CS 60319, 60203 Compiègne Cedex, France.

*Corresponding authors:

- B. Tse Sum Bui; E-mail: jeanne.tse-sum-bui@utc.fr, Tel: +33 344234402, Fax: +33 344203910

- C. Rossi; E-mail: claire.rossi@utc.fr, Tel: +33 344234585, Fax: +33 344203910

\begin{abstract}
Betanin is a natural pigment with significant antioxidant and biological activities currently used as food colorant. The isolation of betanin is problematic due to its instability. In this work, we developed a fast and economic procedure based on molecularly imprinted solid-phase extraction (MISPE) for the selective clean-up of betanin and its stereoisomer isobetanin from beetroot extracts. Dipicolinic acid was used as template for the MIP preparation because of its structural similarity with the chromophore group of betanin. The MISPE procedures were fully optimized allowing the almost complete removal of matrix components such as sugars and proteins, resulting in high extraction recovery of betanin/isobetanin in a single step. Moreover, the whole extraction procedure was performed in environmentally friendly solvents with either ethanol or water. Our MISPE method is very promising for the future development of well-formulated beetroot extract with specified betanin/isobetanin content, ready for food or medicinal use.
\end{abstract}

Keywords: betanin, beetroot extract, dipicolinic acid, molecularly imprinted polymer, solid phase extraction, antioxidants. 


\section{Introduction}

Interest in developing more efficient and selective methods for clean-up and preconcentration of natural-sourced pigments is continuously growing. This is primarily linked to the explosion of the natural food colorant market since some synthetic pigments have been suggested to be involved in child hyperactivity $[1,2]$ and are more and more negatively perceived by the consumers. Moreover, most plant pigments exhibit antioxidant properties which render them highly attractive for applications in the pharmaceutical, cosmetic and nutraceutical areas. For instance, dietary antioxidant pigments such as fatsoluble carotenoids, chlorophylls and curcuminoids and water-soluble anthocyanins have been widely studied for their potential to prevent diseases associated to aging as well as to reduce the risk of cardiovascular disease and cancers [3].

Herein we focus on betanin, a water-soluble pigment belonging to betalains, a class of highly bioavailable natural pigments [4]. The major commercially exploited betalain crop is red beetroot (Beta vulgaris L.), which contains two groups of betalains, the red-violet betacyanins and the yellow-orange betaxanthins of which betanin and vulgaxanthin I (Fig. 1) are the predominant pigments, respectively [5]. Beetroot extract has been approved by the Food and Drug Administration (FDA) and the European Union, as a natural red-violet coloring agent (E162) and is used worldwide for coloring food, beverages, cosmetics and drugs [6,7]. Beetroot extract sold on the market contains a number of different pigments but the predominant colouring principle consists of a number of betacyanins (red) of which betanin accounts for $75-95 \%$ and its C15-epimer, isobetanin 15-45\% [7]. The betanin and isobetanin contents are strongly dependent on beetroot cultivar, farming and storage conditions [8]. Besides their colorant properties, betanin and other betacyanins exert significant antioxidant activity, protecting against oxidative stress both in vitro and in vivo [9,10]. Importantly, other studies have reported their cancer preventive effect and it has been shown that red beet coloring agent E162 reduced significantly the incidence of skin, lung, liver, colon and esophagus tumors in various animal models [11] and in human pancreatic, breast and prostate cancer cell lines [12]. Besides, pure betanin has been demonstrated to 
inhibit tumor growth of several human cancer cell lines in vitro $[13,14]$. Lately, our group has shown that a purified dried beetroot extract containing $80 \%$ of betanin/isobetanin in a ratio $0.6 / 0.4(20 \%$ impurities attributed to proteins) inhibits cancer cell proliferation and viability [15]. Additionally, betacyanins feature high bioavailability $[9,16,17]$ which conditions their health-promoting properties. In fact, very low amounts of unmetabolized betanin/isobetanin $(0.28$ to $0.9 \%)$ of the administered dose was recovered in human urine after consumption of red beet juice. For all the above reasons, a well-formulated beetroot extract with a specified betanin content, appears to be very promising for preventive and therapeutic applications.

Authorized betanin sold on the market is obtained by isolation from red beet. Extraction is conventionally performed in water/alcoholic solutions, due to the hydrophilic nature of betanin [18]. It is worth noting that the stability of betanin poses a great challenge during extraction as variations in temperature (exceeding $50{ }^{\circ} \mathrm{C}$ ), water activity, oxygen, light and $\mathrm{pH}$ (stable between $\mathrm{pH} 3$ to 7) may cause significant degradation [19]. After extraction, purification is carried out by conventional column chromatography such as normal and reversed phase silica [20,21], ion-exchange [21], gel permeation $[9,21,22]$ and polymeric resins $[13,15,20]$, very often followed by a (semi)-preparatory reversed phase HPLC $[9,15]$. However, these methods suffer from a number of disadvantages, such as long processing time, use of non-environmentally friendly organic solvents, betanin degradation during processing or salt introduction in the case of the ion-exchange elution step [21]. Thus, an extended use of betanin as a bioactive compound requires alternative solutions for the efficient clean-up from its natural source.

For this reason, we developed a single step purification method based on molecularly imprinted polymers for the selective clean-up of betanin from crude beetroot extract. Molecularly imprinted polymers are tailor-made biomimetic materials capable of specific recognition towards a target molecule. They are synthesized by co-polymerizing functional and cross-linking monomers in the presence of a molecular template. Subsequent removal of the template leaves complementary binding sites with affinities and specificities comparable to those of natural antibodies. Their molecular recognition 
properties, combined with their high stability, mechanical robustness, low cost and easy synthesis make them extremely attractive as selective capture materials with applications in analytical separations [23,24] and sensing $[25,26]$, among others. Advantageously, the use of MIPs as affinity sorbents allows not only the pre-concentration of target analytes, but also the elimination of matrix components in complex samples [27-29]. Due to their high selectivity, MIPs have been extensively used as sorbents in SPE (namely MISPE) for the extraction of compounds from a large variety of matrices including food [30,31] and of bioactive compounds with antioxidant properties such as curcuminoids and quercetin from vegetal samples $[32,33]$.

Beetroot constitutes quite a complex medium and is mainly composed of carbohydrates, sugars, proteins, vitamins, amino acids and minerals [5], which can interfere with molecular recognition of betanin. Betanin itself cannot be used as template for MIP preparation due to its low stability in general and under polymerization conditions. To circumvent this problem and to avoid eventual interference by betanin bleeding during analysis, we employed a dummy template [27]. Betalamic acid (Fig. 1), the chromophore common to all betalain pigments would have been a good choice but is also chemically very unstable. Therefore, a commercially cheap and stable molecule, dipicolinic acid (DPA) (Fig. 1) was tested. The resulting MIP has a very high specificity for DPA in water since the binding of the corresponding non-imprinted polymer (NIP) was negligible. This polymer was then applied as a MISPE sorbent for betanin/isobetanin extraction. The purity of the sample was confirmed by LC/MS-MS. To the best of our knowledge, this is the first report of a MIP describing the purification of betanin/isobetanin from beetroots. 


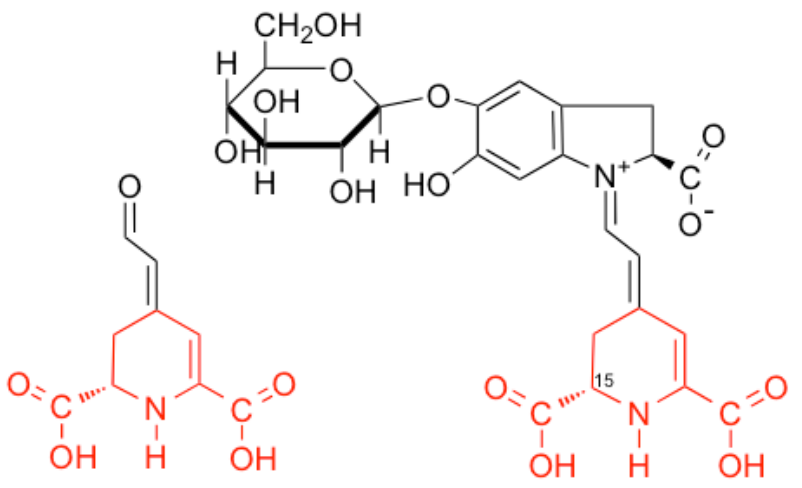

Betalamic acid

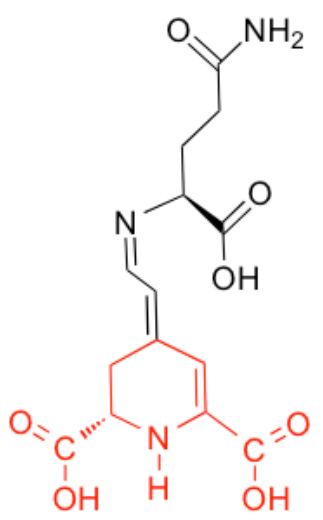

Vulgaxanthin I

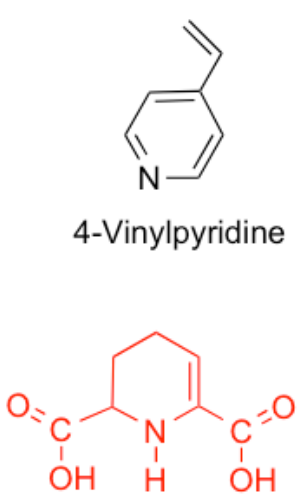

Dipicolinic acid

Fig. 1. Chemical structures of compounds described in this study. *Isobetanin: C15 epimer of betanin.

\section{Experimental}

\subsection{Chemicals}

All chemicals and solvents were of analytical grade and purchased from VWR International (Fontenay sous Bois, France) or Sigma-Aldrich (St-Quentin Fallavier, France), unless mentioned otherwise. LC-MS solvents were purchased from Biosolve chimie (Dieuze, France). The inhibitor, hydroquinone (100 ppm) in 4-vinylpyridine (4-VP, 95\%) was removed by vacuum distillation. Ethylene glycol dimethacrylate (EGDMA) was used as received. Azo-bis-dimethylvaleronitrile (ABDV) was from DuPont Chemicals (Wilmington, USA). Folin Ciocalteau phenol reagent 2N was diluted 2-fold in water prior to use. Water was deionized (resistivity higher than $18.2 \mathrm{M} \Omega \cdot \mathrm{cm}^{-1}$ ) and filtered using a Milli-Q plus unit (Millipore, Molsheim, France).

\subsection{Extraction of pigments from beetroots}

Fresh red beetroots were purchased from a local market. Unpeeled beetroots were finely grated and mixed with ethanol:water (4:1) for extraction of pigments, under continuous mechanical stirring for $1 \mathrm{~h}$ in the dark. Typically $300 \mathrm{~mL}$ of solvent was used per $100 \mathrm{~g}$ of beetroot. The mixture was centrifuged at $30,000 \mathrm{~g}$ for $15 \mathrm{~min}$ and the supernatant was removed to be re-centrifuged once again. The new supernatant was then filtered using a cellulose filter (pore size $2 \mu \mathrm{m}$, Whatman). Ethanol was evaporated 
under reduced pressure and the resulting crude extract was filtered through an hydrophilic polypropylene membrane (pore size $0.2 \mu \mathrm{m}$, Pall Corporation). The crude extract was stored at $-20{ }^{\circ} \mathrm{C}$ and diluted to the required concentration with Milli-Q water just prior use.

\subsection{Characterization of beetroot extract}

Quantifications of betanin/isobetanin, protein and total carbohydrate contents were recorded on a Cary $60 \mathrm{UV}-$ Visible spectrophotometer (Agilent technologies) at $20^{\circ} \mathrm{C}$.

Betanin/isobetanin quantification: The concentration of betanin/isobetanin pigments in all samples was evaluated spectrophotometrically using the multi-component method, which takes into account small amounts of interfering substances, as described by J. H. von Elbe [34]. In a 1-cm path glass cuvette, the absorbance values of the samples (diluted if necessary) are measured at $538 \mathrm{~nm}$ (a) and $600 \mathrm{~nm}$ (c) to calculate the betanin/isobetanin concentration and to correct for small amounts of impurity, respectively. The corrected light absorbance of betanin/isobetanin $(\mathrm{x})$ is calculated as $\mathrm{x}=1.095(\mathrm{a}-\mathrm{c})$ and the concentration of betanin/isobetanin (calculated in terms of betanin) is obtained using an absorptivity value $\left(\mathrm{A}^{1 \%}\right)_{538 \mathrm{~nm}}$ of 1120 and applying the dilution factor. $\mathrm{A}^{1 \%}=1120$ is the extinction coefficient of betanin representing a $1 \%$ solution $(1.0 \mathrm{~g} / 100 \mathrm{~mL})$.

Total carbohydrate quantification: Carbohydrate content was determined according to the DuBois method [35]. Briefly, $500 \mu \mathrm{L}$ of sample (diluted 10-fold with water) was added into $10 \mathrm{~mL}$ glass vials containing $500 \mu \mathrm{L}$ of $5 \%$ phenol solution (99\%) in water. Then, $2 \mathrm{~mL}$ of concentrated sulfuric acid (98\%) was added to the solution. After vortexing, the samples were kept for $30 \mathrm{~min}$ at $90{ }^{\circ} \mathrm{C}$ and then cooled down to $20^{\circ} \mathrm{C}$. The absorbance was recorded at $492 \mathrm{~nm}$ and compared to a calibration curve using glucose as a standard with concentrations varying from $10 \mu \mathrm{g} / \mathrm{mL}$ to $100 \mu \mathrm{g} / \mathrm{mL}$ (Supplementary, Fig. 1A).

Protein quantification: Protein quantification was performed according to the Lowry method [36]. Briefly, $500 \mu \mathrm{L}$ volume of sample was pipetted in $10 \mathrm{~mL}$ glass tubes, then $2.5 \mathrm{~mL}$ of reagent containing $\mathrm{CuSO}_{4}$ 
and $250 \mu \mathrm{L}$ of Folin Ciocalteau phenol reagent solution diluted to $1 \mathrm{~N}$, were added. After agitation, the samples were incubated for $30 \mathrm{~min}$ in the dark. The absorbance was measured at $750 \mathrm{~nm}$. A calibration curve was constructed using bovine serum albumin (BSA) in water as standard with concentrations varying from 50 to $500 \mu \mathrm{g} / \mathrm{mL}$ (Supplementary, Fig. 1B).

\subsection{Preparation of MIPS}

Typically, $0.1 \mathrm{mmol}$ of DPA, $0.4 \mathrm{mmol}$ of 4-VP, $2 \mathrm{mmol}$ of EGDMA and $0.022 \mathrm{mmol}$ of Vazo 52 were dissolved in $4 \mathrm{~mL}$ of methanol/water (4/1) in a glass vial fitted with an airtight septum. The mixture was then purged with nitrogen for 5 min. Polymerization was done overnight in a water bath at $40{ }^{\circ} \mathrm{C}$. The polymers were then transferred to $50 \mathrm{~mL}$ centrifuge tubes and washed under agitation with 2 rounds of methanol/acetic acid (9/1), 2 rounds of $100 \mathrm{mM} \mathrm{NH}_{3}$ (in water)/methanol (7/3), 2 rounds of water and 2 rounds of methanol. They were then dried overnight under vacuum. Non-imprinted polymers (NIPs) were synthesized in the same way but without the addition of the imprinting template. The yield of polymerization was $\sim 70 \%$, affording $300 \mathrm{mg}$ of polymers.

\subsection{Physical characterization of MIPs}

The hydrodynamic size of the polymers was measured by dynamic light scattering (DLS) using a Zetasizer NanoZS (Malvern Instruments Ltd., Worcestershire, UK) at $25{ }^{\circ} \mathrm{C}$. Scanning electron microscopy (SEM) imaging was carried out on a Philips XL30 Field Emission Gun Scanning Electron Microscope (Amsterdam, Netherlands). Polymer particles were sputter coated with gold prior to measurement.

\subsection{Quantification of dipicolinic acid}

DPA was quantified by measuring the luminescence of its chelate with europium ions. Stock solutions of $2.5 \mathrm{mM}$ DPA and $10 \mathrm{mM}$ europium chloride were prepared in ethanol. The DPA stock was diluted 10-fold with water to construct a calibration curve (12.5 - $125 \mu \mathrm{M})$ (Supplementary, Fig. 2); 50 to 
$500 \mu \mathrm{L}$ of DPA was added to $100 \mu \mathrm{L}$ of $\mathrm{EuCl}_{3}$ in $1.5 \mathrm{~mL}$-polypropylene microcentrifuge tubes in the dark, which was completed by water to a volume of $1 \mathrm{~mL}$. The samples were placed in a quartz cuvette for recording of their luminescence emission at $615 \mathrm{~nm}$ using an excitation wavelength of $280 \mathrm{~nm}$, slit 3 nm. All fluorescence measurements were performed on a Fluorolog-3 fluorescence spectrophotometer (Horiba Jobin Yvon, Longjumeau, France) at $20^{\circ} \mathrm{C}$.

\subsection{Equilibrium binding assays}

The imprinted and non-imprinted particles $(20$ or $40 \mathrm{mg} / \mathrm{mL})$ were suspended in methanol/water or water in a sonicating bath. From this stock suspension, polymer concentrations ranging from 0.5 to 16 $\mathrm{mg} / \mathrm{mL}$ were pipetted in separate $1.5 \mathrm{~mL}$-polypropylene microcentrifuge tubes and $25 \mu \mathrm{M}$ of DPA (stock solution of $2.5 \mathrm{mM}$ in ethanol) was added. The final volume was adjusted to $1 \mathrm{~mL}$ with solvent. The samples were incubated overnight at ambient temperature on a tube rotator (SB2, Stuart Scientific). They were then centrifuged at $40000 \mathrm{~g}$ for $45 \mathrm{~min}$ and a $700 \mu \mathrm{L}$ aliquot of the supernatant was withdrawn for analysis. $70 \mu \mathrm{L}$ of $\mathrm{EuCl}_{3}$ (stock solution of $10 \mathrm{mM}$ in ethanol, stored in the dark at $-20{ }^{\circ} \mathrm{C}$ ) was subsequently added to each sample. The samples were placed in a quartz cuvette for recording of their luminescence. The amount of DPA bound to the polymers was calculated by subtracting the amount of unbound DPA from the initial amount of DPA added.

For capacity studies, a fixed amount of polymers at $8 \mathrm{mg} / \mathrm{mL}$ was used and the concentration of DPA varied from 12.5 to $150 \mu \mathrm{M}$ (stock solution of DPA $2.5 \mathrm{mM}$, diluted 10 fold with water to $250 \mu \mathrm{M}$ ). The imprinted and non-imprinted particles $(40 \mathrm{mg} / \mathrm{mL})$ were suspended in water in a sonicating bath and a $200 \mu \mathrm{L}$ aliquot was pipetted in separate $1.5 \mathrm{~mL}$-polypropylene microcentrifuge tubes. The appropriate amount of DPA (50 to $600 \mu \mathrm{L}$ ) was added to each sample. The final volume was adjusted to $1 \mathrm{~mL}$ with water. The samples were incubated overnight at ambient temperature on a tube rotator. They were then centrifuged at 40,000 $\mathrm{g}$ for $45 \mathrm{~min}$ and a $700 \mu \mathrm{L}$ aliquot of the supernatant was withdrawn for analysis. 70 $\mu \mathrm{L}$ of $10 \mathrm{mM}$ of $\mathrm{EuCl}_{3}$ was subsequently added to each sample and the luminescence measured. 
In order to have a hint of whether betanin in beetroot extract will be recognized by the MIP, binding experiments were done before MISPE. $30 \mathrm{mg}$ of MIP or NIP were weighed in $1.5 \mathrm{~mL}$ polypropylene microcentrifuge tubes. $1 \mathrm{~mL}$ of diluted beetroot extract (concentration of betanin/isobetanin $10 \mu \mathrm{g} / \mathrm{mL}$ measured spectrophotometrically, as described in Section 2.3) was added and incubated for $2 \mathrm{~h}$ at $20^{\circ} \mathrm{C}$. They were then centrifuged at $40,000 \mathrm{~g}$ for $45 \mathrm{~min}$ and a $700 \mu \mathrm{L}$ aliquot of the supernatant was withdrawn for analysis. The absorbance was recorded at 538 and $600 \mathrm{~nm}$, as described [34]. The amount of betanin/isobetanin bound to the polymers was calculated by subtracting the amount of unbound analyte from the initial amount of analyte.

\subsection{Evaluation of imprinted materials by SPE}

The MIP capacity for betanin/isobetanin was determined as follows: $60 \mathrm{mg}$ of MIP was slurry packed in a $1 \mathrm{~mL}$ disposable cartridge equipped with $10 \mu \mathrm{m}$ porous polyethylene frits (Sigma-Aldrich, France). After conditioning with $4 \mathrm{~mL}$ water, increasing volume of diluted beetroot extract (betanin/isobetanin 10 $\mu \mathrm{g} / \mathrm{mL}$ ), by fraction of $0.5 \mathrm{~mL}$, was loaded. The flow-through was collected by fractions of $0.5 \mathrm{~mL}$ in separate tubes. The amount of betanin/isobetanin was determined in each fraction by UV-Vis measurements.

For betanin/isobetanin extraction, typically, $60 \mathrm{mg}$ of MIP and NIP were slurry packed in separate 1 $\mathrm{mL}$ disposable cartridges. The cartridges were placed in a Waters SPE 12-vial vacuum manifold connected to a vacuum pump (PC3001 Vario ${ }^{\text {pro, }}$ Vaccuobrand, France). After conditioning the polymers with $4 \mathrm{~mL}$ of water, $2 \mathrm{~mL}$ of diluted beetroot extract $(10 \mu \mathrm{g} / \mathrm{mL}$ of betanin/isobetanin), was loaded. The cartridges were subsequently washed with water $(4 \mathrm{~mL})$ to remove the unretained compounds. Betanin/isobetanin was eluted with $4 \mathrm{~mL}$ of ethanol/acetic acid (100\%) (1/1). All eluted fractions were pooled and immediately lyophilized to remove ethanol and acetic acid. The lyophilized sample was then dissolved in $1 \mathrm{~mL}$ of water and together with the pooled flow-through and washing fractions, were analyzed by spectrophotometry at 538 and $600 \mathrm{~nm}$ to determine the betanin/isobetanin amount and the 
extraction recovery. The columns were regenerated with $10 \mathrm{~mL}$ of a mixture of ethanol/ $\mathrm{HCl}(37 \%), \mathrm{pH}$ 2.0 and washed abundantly with water.

\subsection{HPLC-ESI-MS/MS analysis}

LC-MS/MS was employed for the identification of individual components contained in the beetroot extract. The HPLC system (Infinity 1290, Agilent Technologies, France) was equipped with a diode array detector coupled to a Q-TOF micro hybrid quadrupole time of flight mass spectrometer (Agilent 6538, Agilent Technologies, France). HPLC analyses were performed on a Thermo Scientific Hypersyl C18 reversed phase $(\mathrm{RP})$ column $(150 \times 2.1 \mathrm{~mm}, 3 \mu \mathrm{m}, 100 \mathrm{~A})$. The mobile phase consisted of water $+0.1 \%$ formic acid (eluent A) and acetonitrile (eluent B). The gradient program began with $0 \% \mathrm{~B}$, ramped to $13 \% \mathrm{~B}$ at $21 \mathrm{~min}$, held at $13 \%$ for $4 \mathrm{~min}$, increased to $100 \% \mathrm{~B}$ at $30 \mathrm{~min}$ and was kept constant at $100 \% \mathrm{~B}$ until $35 \mathrm{~min}$. The flow rate was set at $0.4 \mathrm{~mL} / \mathrm{min}$. Detection was monitored at 204, 477 and $538 \mathrm{~nm}$. Diluted beetroot juice, the purified sample from the MIP column and a commercially availaible betanin extract in dextrin were prepared so as to obtain a concentration of betanin/isobetanin $10 \mu \mathrm{g} / \mathrm{mL}$ (spectrophotometrically), for analysis. This amount corresponds to a weighed amount of $50 \mathrm{mg}$ of the commercial extract in $1 \mathrm{~mL}$ water. The injection volume was $10 \mu \mathrm{L}$ for the purified extract and for the others, the volume was adjusted so as to obtain a similar area to the betanin area in the purified extract, for comparison. Under these conditions, the retention times for vulgaxanthin I, betanin and isobetanin were $\sim 4.1 \mathrm{~min}, 12.3$ and 14.6 min respectively. Positive ion electrospay (ESI) mass spectra were acquired by scan mode in the range $\mathrm{m} / \mathrm{z} 100$ to $\mathrm{m} / \mathrm{z} 1200$ at electrospray voltage of $3800 \mathrm{~V}$ and fragmentor voltage of $140 \mathrm{~V}$. Nitrogen was used as the dry gas at a flow rate of $12.0 \mathrm{~L} / \mathrm{min}$ and a pressure of 45.0 psi. The nebulizer temperature was set to $350{ }^{\circ} \mathrm{C}$.

Betanin/isobetanin and vulgaxanthin I structural identities were confirmed by tandem mass spectrometry using a collision energy of $15 \mathrm{eV}$ and $20 \mathrm{eV}$ respectively. 


\section{Results and discussion}

\subsection{Synthesis and evaluation of the binding characteristics of MIP in batch mode}

Dipicolinic acid (pyridine-2,6-dicarboxylic acid) (Fig. 1) has some structural similarity with betanin/isobetanin, is commercially available and cheap; therefore was selected as template for the preparation of our MIP. Betalamic acid (Fig. 1), the structural element of all betalains, though structurally more closely related to the target analytes, could not be used as it is unstable and decomposed easily. DPA is a biomarker of bacterial endospores and MIPs as sensing materials for this molecule in the assessment of sterility of medical instruments, and for problems related to food spoilage and bioterrorism, have been described in the literature. The different MIPs were prepared using metal-chelating monomers synthesized in-house [37], were of unknown water-compatibility [38] or were synthesized using a sol-gel approach at a high temperature [39].

As our MIP had to be highly specific and selective under mild and aqueous conditions, we used one of our well-established protocols commonly employed for MIP synthesis of 2,4-dichlorophenoxyacetic acid (2,4-D) [40], which like DPA, possesses an aromatic ring and an acidic group. Thus, the MIP was prepared by free radical polymerization using 4-VP as functional monomer, EGDMA as crosslinker in a ratio template:monomer:crosslinker of 1:4:20, Vazo 52 as initiator and methanol/water (4/1) as solvent. The volume of solvent used was 8 times more than described in our previous report [40] so as to avoid the obtention of hard bulk particles that needs to be ground and sieved. Instead a compact soft gel which sediments easily was obtained. The MIP and the non-imprinted control polymer NIP were characterized by dynamic light scattering (DLS) analysis and scanning electron microscopy (SEM). Their hydrodynamic size distribution, represented in Supplementary Fig. 3A, shows a mean diameter of $\sim 3 \mu \mathrm{m}$ and $\sim 2 \mu \mathrm{m}$ for the MIP and NIP respectively. The SEM images additionally show that the dry particles appear agglomerated (Supplementary, Fig. 3(B-C)).

The binding behaviour of the polymers towards DPA was investigated in the solvent of synthesis (Supplementary, Fig. 4) and in water (Fig. 2A). DPA was assayed in the presence of $1 \mathrm{mM}$ of europium 
chloride. The europium dipicolinate complex formed was quantified by measuring the luminescence emission of $\mathrm{Eu}^{3+}\left(\lambda_{\mathrm{ex}}=280 \mathrm{~nm}, \lambda_{\mathrm{em}}=615 \mathrm{~nm}\right)$ that results from energy transfer from DPA excited state to the metal ion while the luminescence of $\mathrm{Eu}^{3+}$ alone is weak [41]. The calibration curve of the complex in water is presented in Supplementary Fig. 2.

Equilibrium binding assays in both methanol/water (Supplementary, Fig. 4) and water (Fig. 2A) showed that the MIP is very specific for DPA as no or negligible binding was observed with the NIP, respectively. A synergistic combination of electrostatic and $\pi-\pi$ stacking interactions between the basic and aromatic monomer 4-VP and DPA might be responsible for the strong complex association in a polar environment, just like for 4-VP and 2,4-D [42]. The binding capacity of the MIP for DPA was determined by plotting a graph of B (bound) versus F (free) (Fig. 2B). The data, fitted to a Langmuir model, showed a dissociation constant $\left(\mathrm{K}_{\mathrm{d}}\right)$ of $3.2 \times 10^{-4} \mathrm{M}$ and a maximum binding capacity $\left(\mathrm{B}_{\max }\right)$ of $9.3 \mathrm{nmol} / \mathrm{mg}$ of MIP. These values are similar to those reported for MIP (DPA) prepared using in-house functional monomers [37] and for MIP (2,4-D) using similar conditions as we employed for the preparation of our MIP [43].

Since the MIP was very efficient in specifically capturing dipicolinic acid in aqueous conditions, the next step was to evaluate its recognition properties for betanin/isobetanin. Thus, binding studies were performed with diluted beetroot juice (corresponding to $10 \mu \mathrm{g} / \mathrm{mL}(18.2 \mathrm{nmol} / \mathrm{mL})$ of betanin/isobetanin, measured spectrophotometrically as described in Section 2.3). Fig. 2C shows that there is binding, however with a lower capacity than for DPA; for instance, $32 \mathrm{mg}$ of MIP is needed to bind $14 \mathrm{nmol}$ of betanin/isobetanin (Fig. 2C) whereas only $4 \mathrm{mg}$ is needed to bind $14 \mathrm{nmol}$ DPA (Fig. 2A). Moreover, the MIP binds the target analytes to a higher extent than the NIP, indicating specificity. Thus, we could proceed further in the study of MISPE for the clean-up of betanin/isobetanin in crude beetroot extract. 

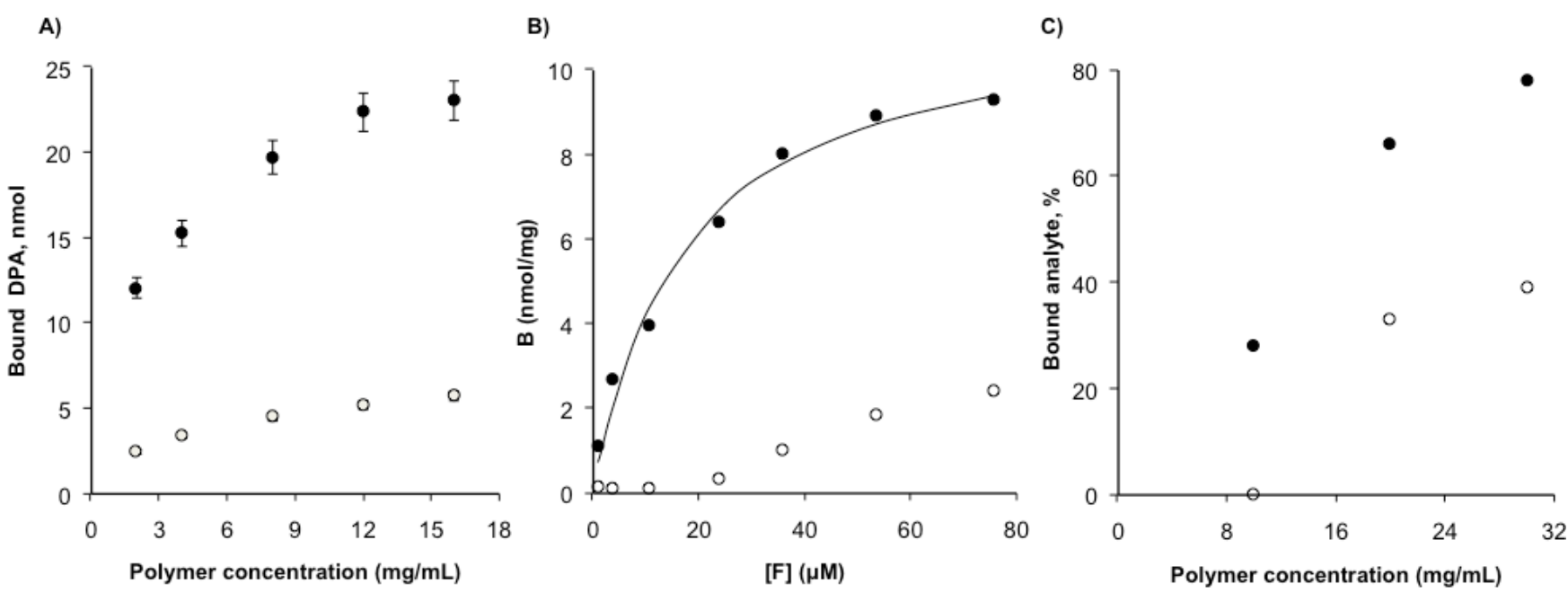

Fig. 2. (A) Equilibrium binding isotherms of MIP (filled circles) and NIP (empty circles) for spiked 25 $\mu \mathrm{M}$ DPA in water. Data are the mean from three independent experiments with three different batches of polymers; (B) Binding capacity of MIP and NIP (8 mg) in $1 \mathrm{~mL}$ of water. The concentration of DPA was varied from $10 \mu \mathrm{M}$ to $150 \mu \mathrm{M}$. B and F represent bound and free DPA, respectively. Data are the mean of two independent experiments; (C) Equilibrium binding isotherms of MIP and NIP for $10 \mu \mathrm{g} / \mathrm{mL}$ (18 $\mathrm{nmol} / \mathrm{mL}$ ) of betanin/isobetanin in beetroot extract. The betanin/isobetanin content was measured spectrophotometrically at 538 and $600 \mathrm{~nm}$, as described in Section 2.3.

\subsection{Solid phase extraction of betanin/isobetanin from crude beetroot extract}

The MIP was first packed in an SPE cartridge so as to determine its binding capacity for betanin/isobetanin. Increasing volumes (by step of $0.5 \mathrm{~mL}$ ) of a diluted beetroot extract containing 10 $\mu \mathrm{g} / \mathrm{mL}$ of betanin/isobetanin were percolated on $60 \mathrm{mg}$ of MIP. Each flow-through fraction was collected and analyzed for betanin/isobetanin content (Supplementary Fig. 5A). Thus, a maximum load of $2 \mathrm{~mL}$ of diluted beetroot extract, corresponding to a maximum pigment loss of 5\% (Supplementary Fig. 5B), was selected to further study the extraction behaviour of betanin/isobetanin on the MIP sorbent.

Subsequently, $60 \mathrm{mg}$ of MIP and NIP were packed in separate SPE cartridges and the conditions of loading, washing and elution were optimized. $2 \mathrm{~mL}$ of diluted beetroot was loaded on the columns. The target analytes (red-violet) as well as the betaxanthins (yellow) were retained by the MIP during the loading as compared to the NIP where all the coloured pigments passed through the column without 
retention. Then, a washing step with $4 \mathrm{~mL}$ of water (by fractions of $1 \mathrm{~mL}$ ) was applied to eliminate the non-retained compounds. All the yellow betaxanthins together with a little amount of the red-violet betacyanins were washed out of the column. Further washings with water did not affect the retention of the red pigments. Fig. 3 shows that the total pigment loss on the MIP does not exceed $20 \pm 5 \%$ while on the NIP, there is a loss of $95 \pm 0.5 \%$ during these two steps. The fact that initially both betaxanthins and betacyanins were retained on the MIP and not at all on the NIP indicates that high-fidelity imprinted sites were present. The two betalains have the same structural backbone as DPA (Fig. 1) and the retention of both is expected. However, during the washings with water, betaxanthins were completely washed out probably due to the difference in polarity between the two betalains [17,21]. Indeed, evidence of the elimination of vulgaxanthin I, the major betaxanthin was confirmed by LC-MS/MS (Section 3.3).

Afterwards, the elution step was optimized taking into account the low stability of the target molecules at pHs values lower than 3 and higher than 7 [19]. To that aim, different elution solvent compositions were tested, namely ethanol, ethanol/acetic acid and ethanol/trifluoroacetic acid at different ratios so as to have different pHs. The best recovery was achieved using ethanol/acetic acid (1/1), $\mathrm{pH} 3.0$. $4 \mathrm{~mL}$ of eluent was applied to ensure maximal recovery of the target molecules. The eluted fractions were pooled and immediately lyophilized to remove ethanol and acetic acid, so as to avoid pigment degradation. The lyophilized sample was then dissolved in $1 \mathrm{~mL}$ of water for analysis. Under these conditions, the extraction recovery for betanin/isobetanin was $80 \pm 5 \%$ for the MIP and $5 \pm 0.5 \%$ for the NIP (Fig. 3), indicating a highly specific retention of the target analytes by the MIP sorbent. The column was washed with ethanol/HCl, $\mathrm{pH} 2.0$ to regenerate the MIP, allowing its reuse for up to 10 times without significant loss of its binding capacity and specificity. 


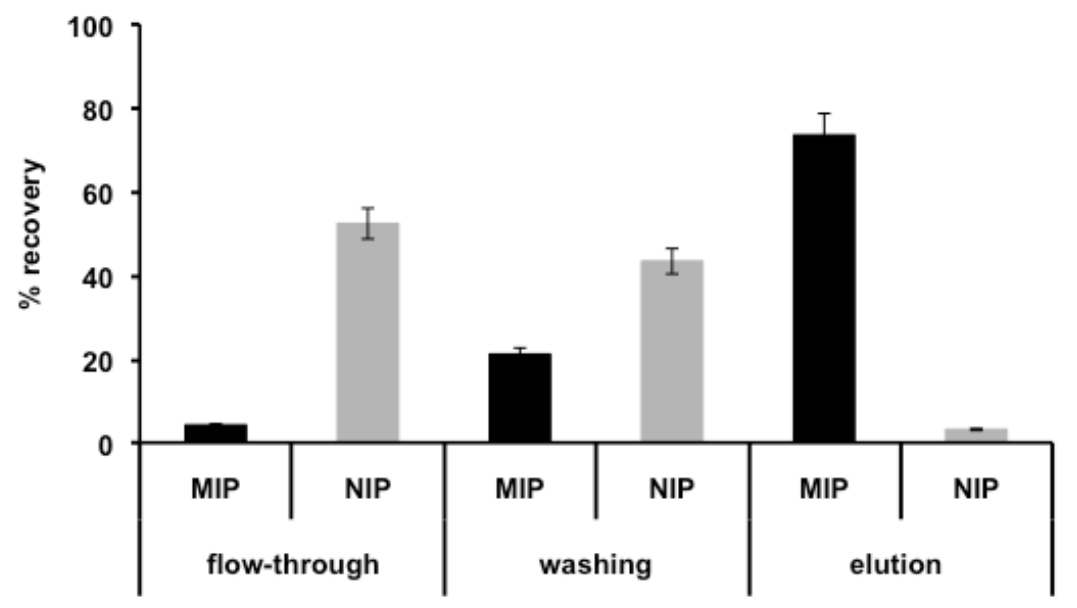

Fig. 3. Extraction recoveries of betanin/isobetanin obtained on $60 \mathrm{mg}$ of polymers. Conditioning with 4 $\mathrm{mL}$ of water; loading of $2 \mathrm{~mL}$ diluted beetroot extract (betanin/isobetanin $10 \mu \mathrm{g} / \mathrm{mL}$ ); washing with $4 \mathrm{~mL}$ water; elution with $4 \mathrm{~mL}$ ethanol/acetic acid (1/1). Data represent the mean of five independent experiments, with 2 different batches of polymers.

\subsection{HPLC/ESI-MS analysis}

The crude and purified extracts were then subjected to RP-HPLC analysis coupled with UV-Vis (204 and $477 \mathrm{~nm}$ ) and electrospray mass spectrometry in positive ion mode for the identification of individual components. Fig. 4B shows that the major components present in the purified sample at both $477 \mathrm{~nm}$ (detection of betacyanins and betaxanthins) and $204 \mathrm{~nm}$ (detection of all pigments and impurities) $[6,13,19]$ were betanin $\left(t_{\mathrm{R}}=12.3 \mathrm{~min},[\mathrm{M}+\mathrm{H}]^{+}=551.1497\right)$ and isobetanin $\left(t_{\mathrm{R}}=14.6 \mathrm{~min},[\mathrm{M}+\mathrm{H}]^{+}=\right.$ $551.1497)$, with extraction window of $50 \mathrm{ppm}$; vulgaxanthin $\mathrm{I}\left(t_{\mathrm{R}} \sim 4.1 \mathrm{~min},[\mathrm{M}+\mathrm{H}]^{+}=340.1139\right)$ was also present as a negligible peak ( $1 \%$ with respect to the areas of betanin/isobetanin). This implies that the MIP was very efficient in removing saccharose (as identified by ESI-MS/MS), the major sugar component in beetroots and vulgaxanthin I, the major pigment of betaxanthins, present in the crude extract (Fig. 4A). For comparison, a commercial betanin sample, sold as a mixture with dextrin, was also analyzed; both the presence of vulgaxanthin I and sugars were detected (Fig. 4C). 


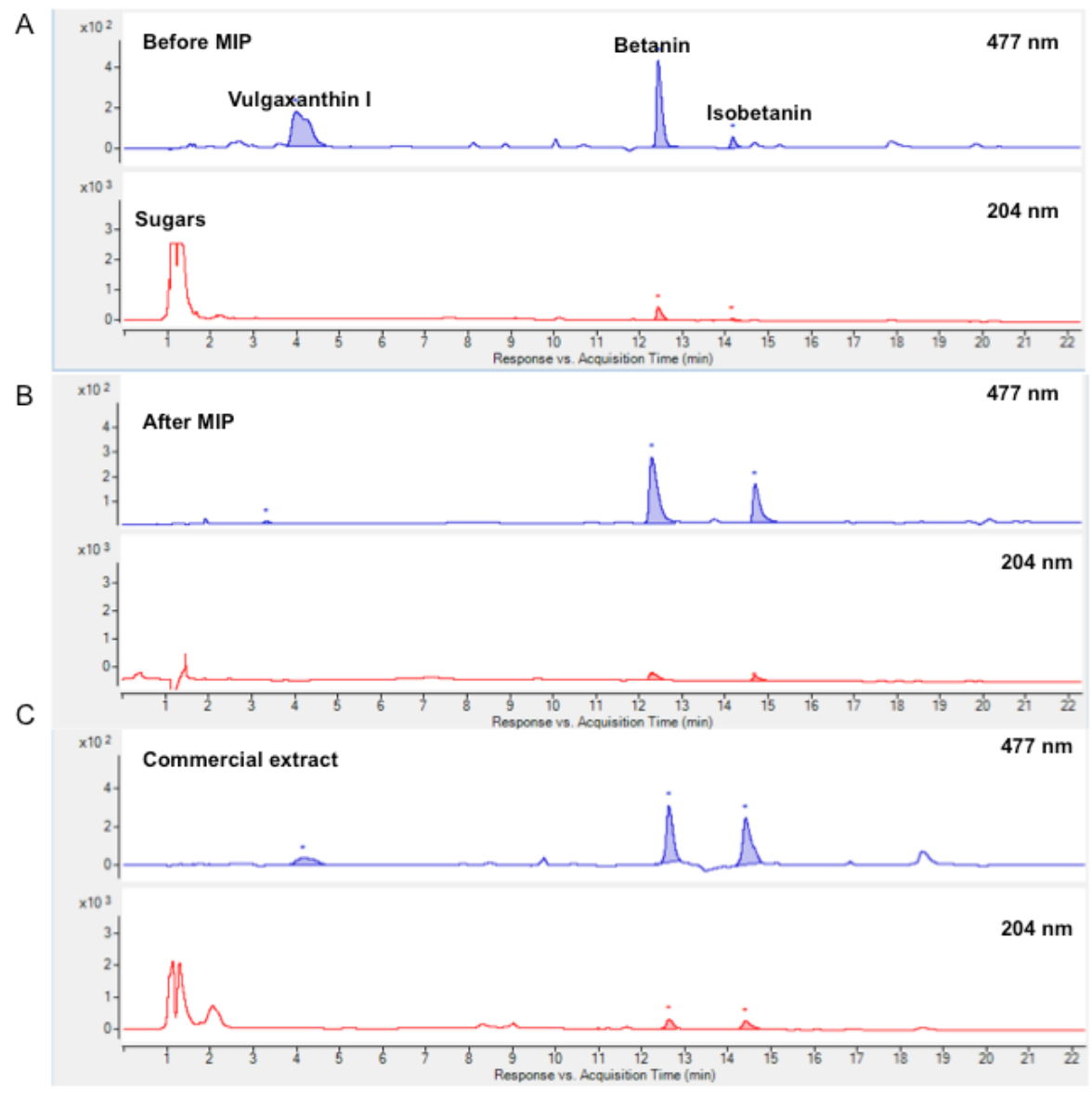

Fig. 4. Representative chromatograms of diluted beetroot extract (A) before and (B) after extraction on MIP and (C) commercial betanin in dextrin. Retention times were attributed by mass spectrometry analysis: sugars (mainly saccharose $\left.\mathrm{m} / \mathrm{z}[\mathrm{M}+\mathrm{Na}]^{+}=365.1054, t_{\mathrm{R}} \sim 1.3 \mathrm{~min}\right)$; vulgaxanthin $\mathrm{I}\left(\mathrm{m} / \mathrm{z}[\mathrm{M}+\mathrm{H}]^{+}\right.$ $\left.=340.1139, t_{\mathrm{R}}=\sim 4.1 \mathrm{~min}\right) ;$ betanin $\left(\mathrm{m} / \mathrm{z}[\mathrm{M}+\mathrm{H}]^{+}=551.1497, t_{\mathrm{R}}=12.3 \mathrm{~min}\right)$ and isobetanin $(\mathrm{m} / \mathrm{z}$ $\left.[\mathrm{M}+\mathrm{H}]^{+}=551.1497, t_{\mathrm{R}}=14.6 \mathrm{~min}\right)$.

Confirmation that the molecules were betanin, isobetanin and vulgaxanthin I was done by tandem mass spectroscopy. The mass spectrum (Fig. 5A) shows the daughter ion produced by fragmentation of the parent ion of $\mathrm{m} / \mathrm{z}$ of 551.1497 assigned to betanin or isobetanin. The fragment ion at the mass charge $(\mathrm{m} / \mathrm{z})$ of 389.0974 indicated that this ion is obtained by glucose loss and corresponds to the protonated aglycone ion, [betanidin $+\mathrm{H}]^{+}$or $[\text {isobetanidin }+\mathrm{H}]^{+}$. The structures between betanin and isobetanin cannot be distinguished by MS/MS, as they differ only by the absolute configuration of the C15 chiral 
center but betanin is slightly more polar, so is less retained as compared to isobetanin on the RP column (Fig. 4) [21,44]. Concerning vulgaxanthin I, the fragment ion at the mass charge $(\mathrm{m} / \mathrm{z})$ of 323.087 corresponds to the daughter ion $\left[\mathrm{M}-\mathrm{NH}_{3}\right]^{+}$produced by fragmentation of the parent ion of $\mathrm{m} / \mathrm{z} 340.1139$, assigned to vulgaxanthin I (Fig. 5B). The other fragment ions are characteristic of vulgaxanthin I, hence proving its identity [44].

A

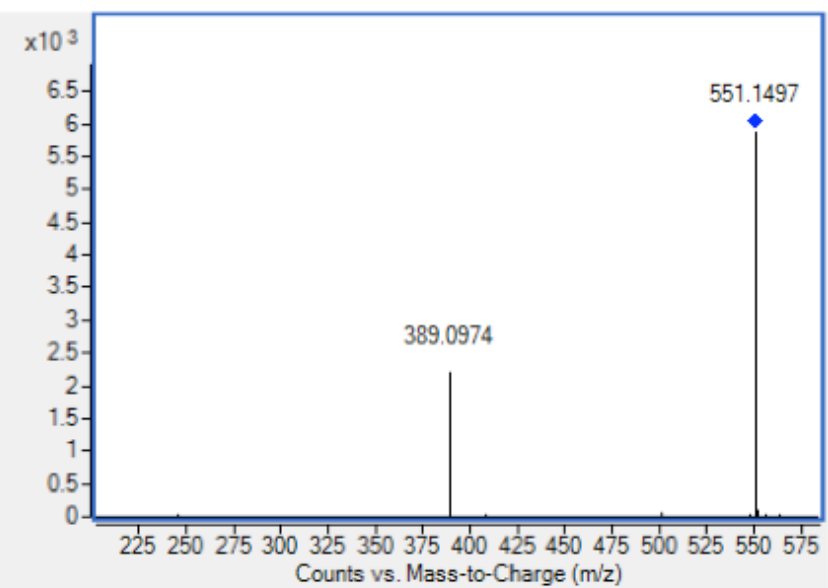

B

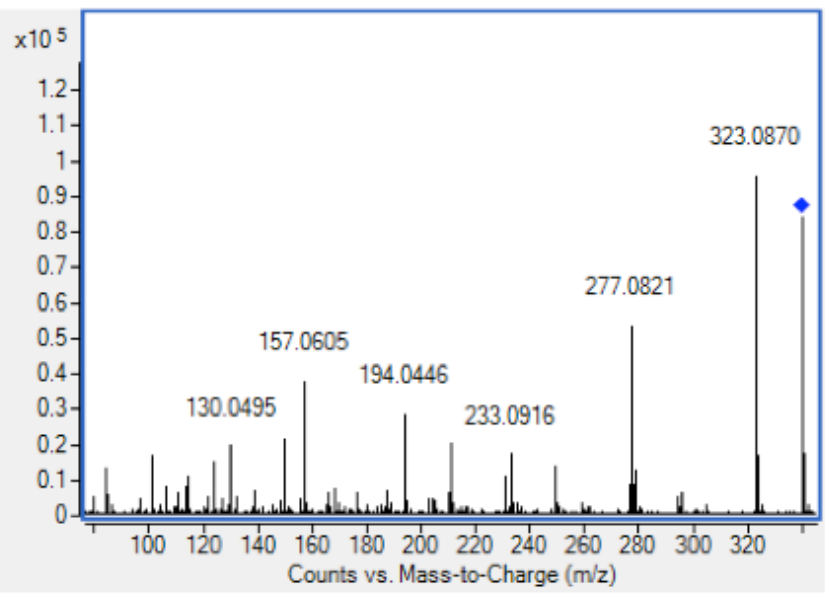

Fig. 5. Positive electrospray tandem mass spectrum of (A) betanin or isobetanin and (B) vulgaxanthin I. The daughter ion of $\mathrm{m} / \mathrm{z} 389.0974$ corresponding to protonated aglycone is obtained by fragmentation of the parent ion of $\mathrm{m} / \mathrm{z} 551.1497$ of betanin or isobetanin. The daughter ion of $\mathrm{m} / \mathrm{z} 323.087$, corresponding to $\left[\mathrm{M}-\mathrm{NH}_{3}\right]^{+}$, is obtained by fragmentation of the parent ion of $\mathrm{m} / \mathrm{z} 340.1139$ of vulgaxanthin I. Collision energy was $15 \mathrm{eV}$ and $20 \mathrm{eV}$ for (A) and (B) respectively.

The HPLC profile of the betanin and isobetanin pigments was also monitored at $538 \mathrm{~nm}$, where they exhibit optimum wavelength response [15,34] (Supplementary, Fig. 6). Based on the peak area values, the betanin/isobetanin ratios of the beetroot extract before and after MIP purification as well as for commercial betanin was calculated and found to be $10.3 \pm 0.2,2.2 \pm 0.1$ and $1.0 \pm 0.1$ respectively. This means that the amount of betanin in the crude, purified and commercial extracts was $91 \%, 69 \%$ and $50 \%$ respectively. These values are consistent with literature data reporting the spontaneous isomerization of betanin to isobetanin during extraction and storage $[19,21]$. The betanin/isobetanin ratio of the purified 
extract is similar to the one obtained previously in our group using $1 \mathrm{~L}$ of Amberlite XAD-16 adsorbent followed by semi-preparative RP-HPLC [15]; thus the MIP could behave as an interesting alternative, in terms of rapidity and economy, to the latter and other conventional non-selective methods [21].

Proteins and sugars are the most abundant constituents in crude beetroot extract [5]. Therefore, the total carbohydrate and protein quantification before and after the MIP column was determined according to the method of DuBois [35] and Lowry [36] respectively. The amount of proteins present in the beetroot extract and the purified fraction was $340 \pm 30 \mu \mathrm{g}$ and $72 \pm 5 \mu \mathrm{g}$ respectively; this means that $21 \%$ of the original proteins was co-eluted with the betanin/isobetanin. For the sugars, there was $731 \pm 60 \mu \mathrm{g}$ present in the crude extract and none in the purified sample as the absorbance measured was lower than the limit of detection (LOD) of the calibration curve (Supplementary Fig. 1A), indicating that they have been totally eliminated on the MIP column and consistent with Fig. 4B. Put together, these results demonstrate for the first time the efficiency of MIPs as selective sorbents for the purification of betanin/isobetanin from its complex natural source.

\section{Conclusions}

We have developed a rapid, eco-friendly and single-step MISPE method for the clean-up of betanin/isobetanin from crude beetroot extract. This method is very economic as all the ingredients used to prepare the MIP are very cheap and moreover the MIP sorbent could be easily regenerated allowing its reuse for up to 10 times without significant loss of its binding capacity and specificity. We believe that this method can be adapted to extract vulgaxanthin I, a potential source of natural yellow pigment from yellow beet which has been scarcely investigated. Indeed vulgaxanthin I was completely separated from betanin/isobetanin. These results pave the way to a wide-scale application of betanin-enriched beetroot extracts for their antioxidant properties in the food and cosmetic areas as well as their antiproliferative activities against tumor cells for biomedical applications. 


\section{Acknowledgments}

We acknowledge funding from the Ministry of National Education Research and Technology (Ph.D. fellowship to S.N), the European Regional Development Fund ERDF and the Region of Picardy (CPER 2007-2013), as well as the federative research structures Condorcet and Cap Santé.

\section{References}

[1] D. McCann, A. Barrett, A. Cooper, D. Crumpler, L. Dalen, K. Grimshaw, E. Kitchin, K. Lok, L. Porteous, E. Prince, E. Sonuga-Barke, J.O. Warner, J. Stevenson, Food additives and hyperactive behaviour in 3-year-old and 8/9-year-old children in the community: a randomised, double-blinded, placebo-controlled trial, The Lancet 370(9598) (2007) 1560-1567.

[2] J.T. Nigg, K. Lewis, T. Edinger, M. Falk, Meta-analysis of attention-deficit/hyperactivity disorder or attention-deficit/hyperactivity disorder symptoms, restriction diet, and synthetic food color additives, J. Am. Acad. Child Adolesc. Psychiatry 51 (2012) 86-97.

[3] Y.J. Surh, Cancer chemoprevention with dietary phytochemicals, Nat. Rev. Cancer 3 (2003) 768-780.

[4] T. Esatbeyoglu, A. E. Wagner, V. B. Schini-Kerth, G. Rimbach, Mol. Nutr. Food Res. 59 (2015), 3647.

[5] B. Neelwarne, S.B. Halagur, Red Beet: An Overview, in: B. Neelwarne (Ed.), Red Beet Biotechnology: Food and Pharmaceutical Applications, Springer, New York, 2012, pp. 1-43.

[6] FDA (US Food and Drug Administration), 2009. Code of Federal Regulations. Title 21. Volume 1. Cite: 21CFR73.260. Sec. 73.260 Vegetable juice. Revised as of April 1, 2009.

[7] EFSA ANS Panel (EFSA Panel on Food Additives and Nutrient Sources added to Food), 2015. Scientific Opinion on the re-evaluation of beetroot red (E 162) as a food additive. EFSA Journal 13 (2015) 4318-4373. doi:10.2903/j.efsa.2015.4318 Available online: www.efsa.europa.eu/efsajournal

[9] J. Kanner, S. Harel, R. Granit, Betalains-a new class of dietary cationized antioxidants, J. Agri. Food Chem. 49 (2001) 5178-5185. 
[10] L. Tesoriere, M. Allegra, C. Gentile, M.A. Livrea, Betacyanins as phenol antioxidants. Chemistry and mechanistic aspects of the lipoperoxyl radical-scavenging activity in solution and liposomes, Free Radical Res. 43 (2009) 706-717.

[11] J.F. Lechner, L.-R. Wang, C.M. Rocha, B.Larue, C.Henry, C.M. McIntyre, K.M. Riedl, S.J. Scwartz, G.D. Stoner, Drinking water with red beetroot food color antagonized esophageal carcinogenesis in Nnitrosomethylbenzylamine-treated rats, J. Med. Food 13 (2010) 733-739.

[12] G.J. Kapadia, G.S. Rao, C. Ramachandra, A. Lida, N. Suzuki, H. Tokuda, Synergistic cytotoxicity of red beetroot (Beta vulgaris L.) extract with doxorubicin in human pancreatic, breast and prostate cancer cell lines, J. Complement Integr. Med. 10 (2013) 1-10.

[13] M. Reddy, R. Alexandro-Lindo, M. Nair, Relative inhibition of lipid peroxidation, cyclooxygenase enzymes, and human tumor cell proliferation by natural food colors, J. Agric. Food Chem. 53 (2005) 9268-9273.

[14] D. Sreekanth, M.K. Arunasree, K.R. Roy, T. Reddy, G.V. Reddy, P. Reddanna, Betanin a betacyanin pigment purified from fruits of Opuntia ficus-indica induces apoptosis in human chronic myeloid leucemia Cell line-K562, Phytomedicine 14 (2007) 739-746.

[15] L. Nowacki, P. Vigneron, L. Rotellini, H. Cazzola, F. Merlier, E. Prost, R. Ralanairina, J.-P. Gadonna, C. Rossi, M. Vayssade, Betanin-Enriched Red Beetroot (Beta vulgaris L.) Extract induces apoptosis and autophagic cell death in MCF-7 cells, Phytother. Res. 12 (2015) 1964-1973.

[16] T. Frank, F.C. Stinzing, R.Carle, I. Bitsch, D. Quaas, G. Straß, R. Bitsch, M. Netzel, Urinary pharmacokinetics of betalains following consumption of red beet juice in healthy humans, Pharmacol. Res. 52 (2005) 290-297.

[17] L. Tesoriere, C. Gentile, F. Angileri, A. Attanzio, M. Tutone, M. Allegra, M.A. Livrea, Transepithelial transport of the betalain pigments indicaxanthin and betanin across Caco-2 cell monolayers and influence of food matrix, Eur. J. Nutr. 52 (2013) 1077-1087. 
[18] F. Delgado-Vargas, A.R Jimenz, O. Paredes-Lopez, Natural pigments: carotenoids, anthocyanins, and betalains-cheracteristics, biosynthesis, processing and stability, Crit. Rev. Food Sci. Nutr. 40 (2000) 173-289.

[19] H. Azeredo, Betalains: properties, sources, applications, and stability- a review, Int. J. Food Sci. Tech. 44 (2009) 2365-2376.

[20] T. Rudrappa, B. Neelwarne, R.G. Aswathanarayana, In situ and ex situ adsorption and recovery of betalains from hairy root cultures of Beta vulgaris, Biotech. Progr. 20 (2004) 777-785.

[21] L.C.P. Gonçalves, M.A.d.S. Trassi, N.B. Lopes, F.A. Dörr, M.T.d.Santos, W.J. Baader, V.X. Oliveira Jr.; E.L. Bastos, A comparative study of the purification of betanin, Food Chem. 131 (2012) 231238.

[22] W. Schliemann, R.W. Joy, A. Komamine, J.W. Metzger, M. Nimtz, V. Wray, D. Strack, Betacyanins from plants and cell cultures of Phytolacca americana, Phytochem. 42 (1996) 1039-1046.

[23] K. Haupt, A.V. Linares, M. Bompart, B. Tse Sum Bui, Molecular imprinted polymers, Top. Curr. Chem. 325 (2012)1-28.

[24] M.J. Whitcombe, N. Kirsch, I.A. Nicholls, Molecular imprinting science and technology: a survey of the literature for the years 2004-2011, J. Mol. Recognit. 27 (2014) 297-401.

[25] X.A. Ton, B. Tse Sum Bui, M. Resmini, P. Bonomi, I. Dika, O. Soppera, K. Haupt. A versatile fluorescence fiber optic sensor based on in-situ polymerized molecularly imprinted microstructures, Angew. Chem. Int. Ed. 52 (2013) 8317-8321.

[26] Molecularly imprinted sensors: overview and applications, S.Li, Y.Ge, S.A. Piletsky, J. Lunec (Eds.), 2012, Elsevier, UK.

[27] B. Tse Sum Bui, K. Haupt, Molecularly imprinted polymers: synthetic receptors in bioanalysis. Anal. Bioanal. Chem. 398 (2010) 2481-2492.

[28] E. Turiel, A. Martín-Esteban, Molecularly imprinted polymers for sample preparation: A review, Anal. Chim. Acta 668 (2010) 87-99. 
[29] R. Kecili, J. Billing, D. Nivhede, B. Sellergren, A. Rees, E. Yilmaz, Fast identification of selective resins for removal of genotoxic aminopyridine impurities via screening of molecularly imprinted polymer libraries, J. Chromatogr. A 1339 (2014) 65-72.

[30] W. Ali, D. Derrien, F.Alix, C. Pérollier, O. Lépine, S. Bayoudh, F. Chapuis-Hugon, V. Pichon, Solid-phase extraction using molecularly imprinted polymers for selective extraction of a mycotoxin in cereals, J. Chromatogr. A 1217 (2010) 6668-6673.

[31] C. Giovannoli, C. Passini, F. Di Nardo, L. Anfossi, C. Baggiani, Determination of ochratoxin A in italian red wines by molecularly imprinted solid phase extraction and HPLC analysis, J. Agric. Food Chem. 62 (2014) 5220-5225.

[32] M. Castro Lopez, M.C. Cela Perez, M. Garcia, J. Vilarino, M. Rodriguez, L. Losada, Preparation, evaluation and characterization of quercetin-molecularly imprinted polymer for preconcentration and clean-up of catechins, Anal. Chim. Acta 721 (2012) 68-78.

[33] M. Wulandari, J. Urraca, A. Descalzo, M. Amran, M. Moreno-Bondi, Molecularly imprinted polymers for cleanup and selective extraction of curcuminoids in medicinal herbal extracts. Anal. Bioanal. Chem. 407 (2015) 803-812.

[34] J.H. von Elbe, Betalains. Current protocols in food analytical chemistry, John Wiley\& Sons Inc., 2001.

[35] M. DuBois, K.A. Gilles, J.K. Hamilton, P.A. Rebers, F. Smith, Colorimetric method for determination of sugars and related substances. Anal. Chem. 28 (1956) 350-356.

[36] O.H. Lowry, N.J. Rosebrough, A.L. Farr, R.J. Randall, Protein measurement with the Folin phenol reagent, J. Biol. Chem. 193 (1951) 265-275.

[37] A. Gültekin, A. Ersöz, N. Y. Sariözlü, A. Denizli, R. Say, Nanosensors having dipicolinic acid imprinted nanoshell for Bacillus cereus spores detection, J. Nanopart. Res. 12 (2010) 2069-2079.

[38] H. Kim, Y. Kim, J.Y. Chang, Preparation of a molecularly imprinted polymer containing europium(III) ions for luminescent sensing, J. Polym. Sci Part A : Polym. Chem. 50 (2012) 4990-4994. 
[39] C. Smith, J. Anderson, J. Edwards, K. Kam, In situ surface-etched bacterial spore detection using dipicolinic acid-europium-silica nanoparticle bioreporters. Appl. Spectrosc. 65 (2011) 866-875.

[40] K. Haupt, A. Dzgoev, K. Mosbach, Assay system for the herbicide 2, 4-dichlorophenoxyacetic acid using a molecularly imprinted polymer as an artificial recognition element, Anal. Chem. 70 (1998) 628631.

[41] J. Zdunek, E. Benito-Pena, A. Linares, A. Falcimaigne-Cordin, G. Orellana, K. Haupt, M.C. Moreno-Bondi, Surface-imprinted nanofilaments for europium-amplified luminescent detection of fluoroquinolone antibiotics, Chem. Eur. J. 19 (2013) 10209-10216.

[42] J. O’Mahony, A. Molinelli, K. Nolan, M.R. Smyth, R. Mizaikoff, Towards the rational development of molecularly imprinted polymers: $1 \mathrm{H}$ NMR studies on hydrophobicity and ion-pair interactions as driving forces for selectivity, Biosens. Bioelectron. 20 (2005) 1884-1893.

[43] J. Li, B. Zu, Y. Zhang, X. Guo, H. Zhang, One-pot synthesis of surface-functionalized molecularly imprinted polymer microspheres by iniferter-induced "living”, radical precipitation polymerization, J. Polym. Sci. Part A: Polym. Chem. 48 (2010) 3217-3228.

[44] I. Račkauskienè, A. Pukalskas, P.R. Venskutonis, A. Fiore, A.D. Troise, V. Fogliano, Effects of beetroot (Beta vulgaris) preparations on the Maillard reaction products in milk and meat-protein model systems, Food Res. Int. 70 (2015) 31-39. 


\section{FIGURE CAPTIONS}

Fig. 1. Chemical structures of compounds described in this study. *Isobetanin: C15 epimer of betanin.

Fig. 2. (A) Equilibrium binding isotherms of MIP (filled circles) and NIP (empty circles) for spiked 25 $\mu \mathrm{M}$ DPA in water. Data are the mean from three independent experiments with three different batches of polymers; (B) Binding capacity of MIP and NIP (8 mg) in $1 \mathrm{~mL}$ of water. The concentration of DPA was varied from $10 \mu \mathrm{M}$ to $150 \mu \mathrm{M}$. B and F represent bound and free DPA, respectively. Data are the mean of two independent experiments; (C) Equilibrium binding isotherms of MIP and NIP for $10 \mu \mathrm{g} / \mathrm{mL}(18$ $\mathrm{nmol} / \mathrm{mL}$ ) of betanin/isobetanin in beetroot extract. The betanin/isobetanin content was measured spectrophotometrically at 538 and $600 \mathrm{~nm}$, as described in Section 2.3.

Fig. 3. Extraction recoveries of betanin/isobetanin obtained on $60 \mathrm{mg}$ of polymers. Conditioning with 4 $\mathrm{mL}$ of water; loading of $2 \mathrm{~mL}$ diluted beetroot extract (betanin/isobetanin $10 \mu \mathrm{g} / \mathrm{mL}$ ); washing with $4 \mathrm{~mL}$ water; elution with $4 \mathrm{~mL}$ ethanol/acetic acid (1/1). Data represent the mean of five independent experiments, with 2 different batches of polymers.

Fig. 4. Representative chromatograms of diluted beetroot extract (A) before and (B) after extraction on MIP and (C) commercial betanin in dextrin. Retention times were attributed by mass spectrometry analysis: sugars (mainly saccharose $\left.\mathrm{m} / \mathrm{z}[\mathrm{M}+\mathrm{Na}]^{+}=365.1054, t_{\mathrm{R}} \sim 1.3 \mathrm{~min}\right)$; vulgaxanthin $\mathrm{I}\left(\mathrm{m} / \mathrm{z}[\mathrm{M}+\mathrm{H}]^{+}\right.$ $\left.=340.1139, t_{\mathrm{R}}=\sim 4.1 \mathrm{~min}\right) ;$ betanin $\left(\mathrm{m} / \mathrm{z}[\mathrm{M}+\mathrm{H}]^{+}=551.1497, t_{\mathrm{R}}=12.3 \mathrm{~min}\right)$ and isobetanin $(\mathrm{m} / \mathrm{z}$ $\left.[\mathrm{M}+\mathrm{H}]^{+}=551.1497, t_{\mathrm{R}}=14.6 \mathrm{~min}\right)$.

Fig. 5. Positive electrospray tandem mass spectrum of (A) betanin or isobetanin and (B) vulgaxanthin I. The daughter ion of $\mathrm{m} / \mathrm{z} 389.0974$ corresponding to protonated aglycone is obtained by fragmentation of the parent ion of $\mathrm{m} / \mathrm{z} 551.1497$ of betanin or isobetanin. The daughter ion of $\mathrm{m} / \mathrm{z} 323.087$, corresponding to $\left[\mathrm{M}-\mathrm{NH}_{3}\right]^{+}$, is obtained by fragmentation of the parent ion of $\mathrm{m} / \mathrm{z} 340.1139$ of vulgaxanthin I. Collision energy was $15 \mathrm{eV}$ and $20 \mathrm{eV}$ for (A) and (B) respectively. 